\title{
Contribution à la connaissance des Cladocères néotropicaux : redescription de Leydigiopsis ornata Daday, 1905. (Crustacea, Cladocera).
}

\author{
J. Reyl \\ E. Vasquez ${ }^{2}$ \\ Mots clés : Taxonomie, Cladocera, redescription, Venezuela.
}

La femelle parthénogénétique de Leydigiopsis omata Daday, 1905 est redécrite. Des données morphologiques complémentaires incluant l'étude détaillé des appendices thoraciques sont fournies. La femelle éphippiale et le mâle, inconnus jusqu'ici, sont également décrits. Les caractères morphologiques différentiels des $\mathbf{4}$ espèces du genre sont discutés.

A contribution to the study of neotropical cladocerans : a re-description of Leydigiopsis ornata Daday, 1905 (Crustacea, Cladocera).

Keywords : Taxonomy, Cladocera, redescription, Venezuela.

The parthenogenetic female of Leydigiopsis omata Daday 1905 is re-described. Complementary morphological data ara provided, included a detailed study of the thoracic appendages. Descriptions are also given of the sexual female (produ. cing ephippia) and male, these being unknown until now. The morphological characters separating the four species in the genus are discussed.

\section{1. - Introduction}

Le genre néotropical Leydigiopsis contient à ce jour 4 espèces :

Leydigiopsis curvirostris Sars, 1901, espèce générotype décrite des environs de Sao Paulo (Brésil) : L. megalops Sars, 1901, également décrite de la même localité ;

L. ornata Daday, 1905, du Paraguay;

L. brevirostris Brehm, 1938, du Brésil (Lagoa do Carro; Rio Jaboatao).

Le travail de Sars (1901) nous fournit une bonne documentation sur Leydigiopsis curvirostris (étude du mâle et de la femelle parthénogénétique). Dans ce même travail, Sars décrit aussi la femelle parthénogénétique de $L$. megalops.

$L$. brevirostris, également connue par la femelle parthénogénétique, a été récemment réétudièe en détail par Valdivia-Villar (1984).

Pour L. omata, connue jusqu'ici seulement par la $\$$ parthénogénétique, nous disposons de la description de Daday (1905) illustrée par 3 figures (habitus, région antérieure et postabdomen). A celle-ci s'ajoutent

1. Laboratoire d'Hydrobiologie. UA CNRS 695, Université Paul Sabatier, 118, route de Narbonne, 31062 Toulouse Cédex, France. 2. Fundaciòn La Salle de Ciencias Naturales. Apdo 51, San Felix, Edo Bolivar, Venezuela. quelques données ultérieures de la littérature (Brehm 1937, Martinez de Ferrato 1966, Goulden 1966) qui n'apportent pas de renseignements complémentaires, excepté la structure des pores céphaliques décrite par Goulden (op. cit.).

Au cours de l'examen de matériel pélagique et littoral de divers corps d'eau du bassin moyen de l'Orénoque (Rey \& Vasquez 1986), nous avons eu l'occasion d"étudier plusieurs spécimens de Leydigiopsis omata dont 19 ephippiale et $1 \%$, inconnus jusqu'ici. La description de ces deux catégories d'individus ainsi qu'une description plus documentée de la $\&$ parthénogénétique comprenant l'étude morphologique détaillée des appendices thoraciques non décrits à ce jour, font l'objel du présent travail.

\section{2. - Leydigiopsis ornata Daday, 1905.}

(PI. I. fig. 1-14 ; Pl. II, fig. 1-7 ; Pl. III, fig. 1-8).

Leydigiopsis omata Daday, 1905 : pp. 187-188, Pl. 12, fig. 1-3 ; Brehm 1937 : pp. 498, 500, 510,511, fig. 6-7: Martinez de Ferrato 1966 : p. 400, Pl. 2, fig. 1-2 ; Goulden 1966 ; Smirnov 1971 : pp. 625-628, fig, 655, 658 ; Zoppi de Roa (sous presse).

Leydigiopsis curvirostris Brehm \& Thomsen, 1936: pp. 211, 213, 217. (synonymie in Smirnov, 1971 : p. 628). 


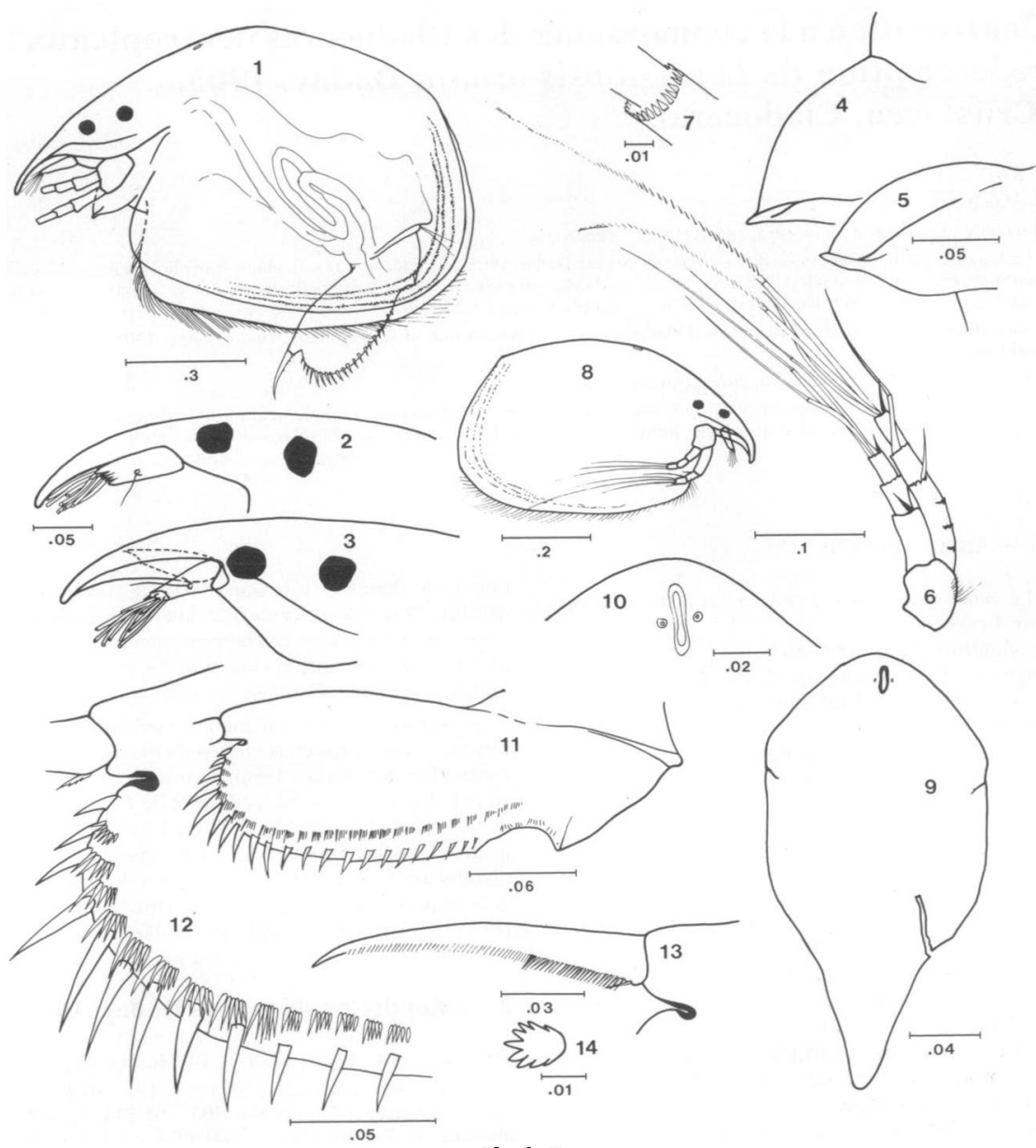

Planche I

Fig. 1 à 14 : Leydigiopsis ornata Daday, 1905. Femelle parthénogénétique. 1 : vue générale latérale. 2 et $3:$ région céphaljque an térieure. 4 et $5:$ labre. $6:$ antenne. $7:$ couronne de sétules distales des articles antennaires. $8:$ longueur relative des soies antennaires et de la carapace. $9:$ bouclier céphalique. $10:$ pores céphaliques. $11:$ postabdomen. $12:$ partie terminale du postabdomen. 13 : griffe postabdominale. 14 : épine basale de la griffe postabdominale en vue dorsale. 
Localité typique : Daday (1905) signale sans mention particulière : Curuzu-Chica, bras mort du fleuve Paraguay; la Lagune Estia Postillon: Paso Barreto, Banado, sur la rive du fleuve Aquidaban.

\section{1. - Matériel examiné}

Tous les spécimens étudiés proviennent de la zone littorale à macrophytes de 2 lacs du système d'inondation de l'Orénoque:

- Lac Playa Blanca-12.3.85.-(Eichhomia crasipes) - 22 individus : 20 femelles parthénogènétiques dont 11 \& 9 non ovigères $(575 \mu$ à $980 \mu)$ et 9 \% 9 ovigères $(760 \mu$ à $870 \mu) ; 1$ éphippiale $(910 \mu) ; 1$ o $(700 \mu)$.

- Lac Lagoven - 5 femelles parthénogénétiques.

20.4.83. (E. crassipes) : 1 ᄋ $(520 \mu)$;

7.9.84. (Nuphar sp., Salvinia sp.): $4 \%$ $\%(520 \mu$ à $755 \mu) ; 1$ q ovigère $(900 \mu)$.

Tous les spécimens ont été montés à la glycérine. La terminologie utilisée se réfère à Fryer $(1963,1968)$ ct Smirnov (1971).

\section{2. - Description}

2.2.1. - Femelle parthénogénétique (Pl. I, fig. 1-14) Carapace (Pl. I : 1 ).

Couleur brun clair, jaune. Forme quadrangulaire à angle postéro-dorsal et postéro-ventral arrondis. Surface des valves présentant 3 à 4 rangées submarginales de granulations s'ordonnant parallèlement aux bords ventral et postérieur des valves, parfois difficiles à voir chez certains individus. Contrairement aux figures de Daday (1905, fig. 1, pl. 12) et de Martinez de Ferrato (1966, fig. 1, pl. 2), la sétation du bord ventral des valves n'est pas uniforme mais représentée par 3 groupes de soies : 24 à 25 longues soies antéroventrales dont les 7 à 8 soies postérieures sont les plus longues, suivies de 10 à 12 soies courtes arrivant environ au milieu de la marge ventrale et, dans la moitié postérieure, 30 à 32 soies de longueur d'abord croissante jusqu'à l'angle postéro-ventral, ensuite rapidement décroissante en direction postéro-dorsale. Ces soies, bisétulées, sont insérées sur une partie basilaire. plus développée dans la région postérieure des valves, portant 2 épines de longueur inégale qui encadrent la base de la soie (Pl. III : 3 ).

Tête (PI. I : 2-5, 9, 10).

Rostre long. Le labre est caractéristique par sa forme triangulaire et son aspect très fortement caréné chez tous les spécimens étudiès. L'apex saille nettement sur le bord antérieur convexe (fig. 1, 4, 5). Bien que dans sa description originale Daday note * Der Lippenanhang ist kegelförmig, der Vorderrand schwach rundet, glatt, das untere Ende spitzig ", cette structure n'est pas figurée dans son travail de 1905 (Tab. XII, fig. 1, 2) ni, dans les travaux ultérieurs.

L'œil et l'ocelle sont arrondis, de taille subégale, l'ocelle plus près de l'œil que de la pointe du rostre. Les pores céphaliques principaux se présentent sous forme d'un canal allongé, comme décrit par Goulden 1966 a et $b$ pour Leydigiopsis megalops et $L$ o omata respectivement. Les 2 pores secondaires latéraux punctiformes sont situés environ à midistance des pores principaux. (fig. 9, 10). Distance postpore (pore postérieur bord postérieur du bouclier céphalique) environ la moitié de la distance interpores. Rapport largeur-longueur du bouclier céphalique : 0,49 .

Antennule (PI. I : 2, 3)

Partie distale tronquée obliquement. 8 à 9 papilles sensorielles de longueur inégale, arrivant environ à la pointe du rostre. Soie sensorielle insérée à mi-longueur de l'antennule sur un petit tubercule.

Postabdomen (Pl. I : 11-14)

Long, élargi dans sa partie terminale, largement arrondi à l'angle dorso-distal, se creusant à la base de la griffe terminale en une depression fortement chitinisée. Angle préanal proéminent. Dépression anale relativement profonde, bordée par 3 à 4 groupes de courtes soies. 21 à 22 denticules marginaux aculéiformes dont 15 à 16 bien développés, les plus grands situés à l'angle dorso-distal ; 25 à 27 fascicules latéraux de soies spinuleuses dont la distale, plus longue et large, dépasse le bord du postabdomen dans la région dorso-distale.

La griffe terminale est longue, légèrement recour. bée à son extrémité. Elle présente dans le tiers proximal une rangée de délicates sétules, la sétule distale plus forte que les autres, suivie d'une fine ciliature. L'épine basale, courte, a un aspect multifide en vue latérale. Vue par dessous (dorsalement), elle est courte, large et fasciculée. (fig. 14).

Antenne (Pl. I: 6, 7, 8)

Bien développée. La grande soie terminale de l'endopode un peu moins longue que chez L. curvirostris, 
atteint seulement le tiers postérieur de la carapace. (fig. 8).

Formule antennaire: $0(1)$. $0(0) .3(1) . / 1(0)$. 1(0. 3(1).

La soie proximale de l'endopode est très courte. Les 3 soies terminales de la même branche sont de grosseur et de longueur inégales. Une de ces 3 soies, relativement courte, a environ la même longueur que la soie du $2^{\circ}$ article de l'endopode. Les 2 autres. plus grosses et très longues, sont également plus longues que les 3 soies de l'exopode, et leur segment distal est armé non de sétules, comme chez les autres soies antennaires, mais de courtes petites spinules, plus épaisses au niveau de la plus grande soie (fig. 6).

Epine terminale de l'endopode plus longue que l'épine terminale de l'exopode mais chacune d'elles aussi longue que l'article qui la porte. Le premier article de l'endopode porte 3 rangées de soies courtes et le basipode, un groupe de soies longues dans sa partie médiane.

En outre, chaque article des 2 branches antennaires porte distalement une couronne de soies courtes, épaisses et triangulaires ( $f \mathrm{ig} .7$ ).

Appendices thoraciques (Pl. II, fig. 1-5)

Patte thoracique I (Pl. II : 1, 1a)

Forme rectangulaire en vue latérale. La partie antérieure porte 3 groupes de sétules: 1 groupe antéro-dorsal et 1 rangée antéro-ventrale de sétules de taille moyenne, et 10 à 11 longues sétules réparties sur toute la face antérieure du corps de l'endite. 2 soies glabres, unciformes (ejector hooks) sont insérées au 1/4 antérieur de l'appendice.

Le lobe postérieur de la branche interne de l'endite présente 5 soies dont 2 sont plus longues, et le lobe antérieur, 4 soies de taille subégale.

La branche externe de l'endite porte 3 soies unisétulées : soie 1, courte, en crochet, soies 2 et 3 relativement grêles et longues. La soie 1 est courtement sétulée sur ses $2 / 3$ distals, la soie intermédiaire également sétulée sur ses $2 / 3$ distals est armée de sétules de longueur movenne et de taille décroissante distalement. La soie 3 garnie de sétules plus courtes sur un peu plus de sa moitié distale, est nettement plus développée, presque aussi longue que la soie de l'exopodite, elle-même grêle et finement ciliée.
Patte thoracique II (Pl. II : 2)

Exopodite réduit, digitiforme, portant une touffe de fines et longues sétules. Endite à 8 soies rigides, bisegmentées, les 6 soies postérieures d'autant plus courtes, larges et fortement sétulées qu'elles se rapprochent de la gnathobase. Face postérieure de la gnathobase avec un coussinet de nombreuses et courtes sétules. Filtre gnathobasique à 7 soies, 6 soies longues et glabres, la $7^{\circ}$ soie (la plus postérieure) légèrement plus courte et un peu à l'écart des autres, paraissant porter quelques fines sétules. Cette soie pourrait correspondre à la "sweeping seta " de Fryer (1968), fonctionnellement en continuité avec le coussinet de sétules qui arme la face postérieure de la gnathobase.

Patte thoracique III (PI. II : 3)

Exopodite subrectangulaire à 7 soies : 5 soies de longueur inégale, dirigées postérieurement et 2 soies antéro-dorsales fortement divergentes dont la plus antérieure est un peu plus de $\mathbf{2}$ fois plus longue que l'autre. Ces 2 soies, de même que la grande soie postéro-dorsale et les 2 soies postérieures intermédiaires, réduites, sont garnies bilatéralement de fines et souples sétules de taille moyenne. Les 2 soies postéro-ventrales, par contre, portent des sétules courtes et raides.

Endite à 8 soies : 3 fortes soies distales en crochet et un groupe de 5 soies plus courtes situées au niveau de la gnathobase. Entre ces 2 groupes de soies, se trouve un tubercule plus ou moins conique (sensille ?). Extrémité de la gnathobase formant 2 saillies et portant 1 soie coudée. Filtre gnathobasique formé de 7 soies glabres et très longues.

Patte thoracique IV (PI. II : 4, 4a)

Exopodite quadrangulaire à 6 soies. Les 2 soies postéro-ventrales sont un peu plus courtes et à sétulation réduite comme pour la P III. Les 4 autres soies, de longueur subégale, sont armées de sétules souples et fines.

Endite : 7 soies de longueur et de structure différentes et 4 soies situées du côté extérieur de l'endite. Filtre gnathobasique à 5 longues soies glabres mais bien moins longues que les soies gnathobasiques de la patte thoracique III.

Patte thoracique V (Pl. II : 5)

Exopodite grand, subovale, à 4 soies : 3 soies regroupées postérieurement et 1 soie dorsale plus 


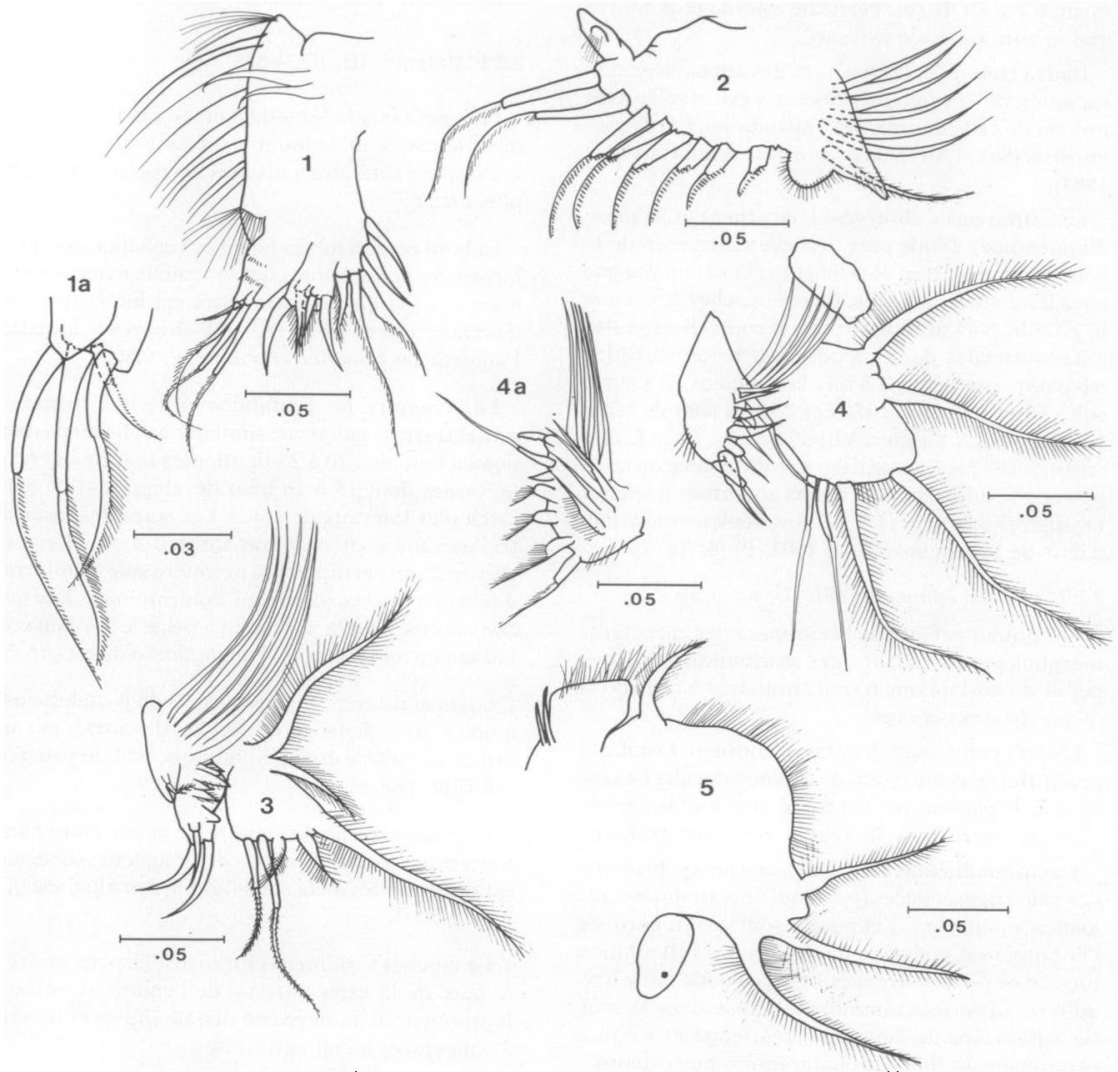

Planche II

Fig. 1 à 5 : Leydigiopsis omata Daday, 1905. Appendices thoraciques. 1 : patte thoracique I. 1 a : branche externe de l'endite et exopodite de la patte thoracique I. 2 : patte thoracique II. 3 : patte thoracique III. $4:$ patte thoracique IV. $4 a$ : endite de la patte thoracique IV. 5 : patte thoracique V. 
courte, toutes les 4 armées de sétules souples et fines. Bord de l'exopodite également sétulé.

Endite réduit, quadrangulaire, sétulé marginalement, à 2 soies, la soie verticale interne plus courte que la soie verticale externe.

Dans l'ensemble, la structure des appendices thoraciques de Leydigiopsis ornata s'est révélée très proche de celle des mèmes appendices chez $L$. cur. virostris (Sars 1901) et L. brevirostris (Valdivia-Villar 1984).

Les différences observées concernent la $3^{\circ}$ paire d'appendices. D'une part, les soies filtrantes de la gnathobase sont bien plus longues chez $L$. omata que chez les 2 autres espèces. De même, chez $L$. omata, la soie la plus antérieure des 2 soies divergentes antéro-dorsales de l'exopodite est nettement plus développée (presque 2.5 fois la longueur de l'autre soie). Chez $L$. brevirostris, ces 2 soies sont de taille subégale (Pl. 2, Valdivia-Villar, op. cit.). Chez L. cur. virostris ces 2 soies montrent une différence de taille mais cette différence est moins accentuée que chez Leydigiopsis ornata (1,5 à 2 fois seulement la lon. gueur de l'autre soie (Sars 1901, Pl. 8, fig. 7).

\subsubsection{Femelle éphippiale (Pl. III, fig. 1-3)}

Le contour général de la carapace, les caractères morphologiques du labre, des antennules, des antennes et du postabdomen sont similaires à ceux de la q parthénogénétique.

L'éphippium, monoloculaire, contient 1 œuf qui apparaît légèrement ovoïde en vue latérale. Le contour de l'éphippie, net sur à peu près tout son pourtour, se perd dans la région inféro-postérieure.

La carène dorsale, large, bien chitinisée, présente des rides transversales (fig. 2) qui se poursuivent aux angles antéro-dorsal et postéro-dorsal. La paroi de l'éphippie est couverte d'une épaisse réticulation formée de petits polygones juxtaposés, de taille irrégulière. Cette réticulation, accentuée dans la moitié supérieure de l'éphippie, s'atténue et s'efface complètement dans la région inféro-postérieure.

La seule observation concernant les femelles éphippiales est celle de Sars (1901) qui note, à propos de Leydigiopsis curvirostris, le caractère peu net de l'éphippie "resting ova were produced... accomDanied by a slight change in the upper part of the carapace which also, in such cases, assumed a somewhat darker line, though no true ephippium was formed .
Dans le cas présent, la structure observée n'offre pas d'ambiguité. Le contour de l'éphippium, la structure monoculaire, la paroi à réticulation fortement sclérotinisée décrivent une véritable éphippie.

\subsubsection{Mầle (Pl. III, fig. 4-8)}

Plus petit que la femelle comme c'est habituellement le cas, le mâle montre un contour général de la carapace similaire mais avec un rostre nettement plus court.

Le bord postérieur du bouclier céphalique est plus largement arrondi que chez la femelle mais, comme chez la $Q$, la distance postpore est inférieure à la distance interpores. (fig. 4). Rapport largeurlongueur du bouclier céphalique : 0,56.

La structure du postabdomen (taille, forme et armature) est également similaire à celle du postabdomen femelle : 20 à 21 denticules marginaux aculéiformes dont 15 à 16 bien développés et 26 à 27 fascicules latéraux de soies. Les soies des fascicules latéraux sont toutefois un peu moins longues chez le $\sigma$, en particulier la première soie spiniforme de chacun des fascicules qui, contrairement à ce que l'on observe chez la $\&$, atteint à peine le bord du postabdomen au niveau de l'angle dorso-distal (fig. 5).

Le canal déférent s'ouvre aux 2/3 du postabdomen, à une courte distance de son bord ventral, par un orifice circulaire dont les contours sont un peu plus sclérifiés (/ig. 6).

La rame externe de l'endite de la première patte thoracique porte 3 soies de longueur subégale, caractéristiques de la sous-famille des Aloninae (fig. 7).

Le crochet fortement chitinisé (clasper), inséré à la base de la rame externe de l'endite, se rétrécit légèrement dans sa partie distale ( $f$ ig. 8 ) et mont re 3 cannelures à son extrémité.

2 touffes distinctes de sétules sont insérées dans la région antéro-dorsale de l'endite. De nombreuses autres sétules raides et serrées garnissent la région antéro-ventrale, mais les longues sétules réparties sur la face antérieure de l'endite femelle sont absentes chez le mâle. Comme chez la femelle 2 soies unciformes, glabres, se situent au quart antérieur de l'appendice. 


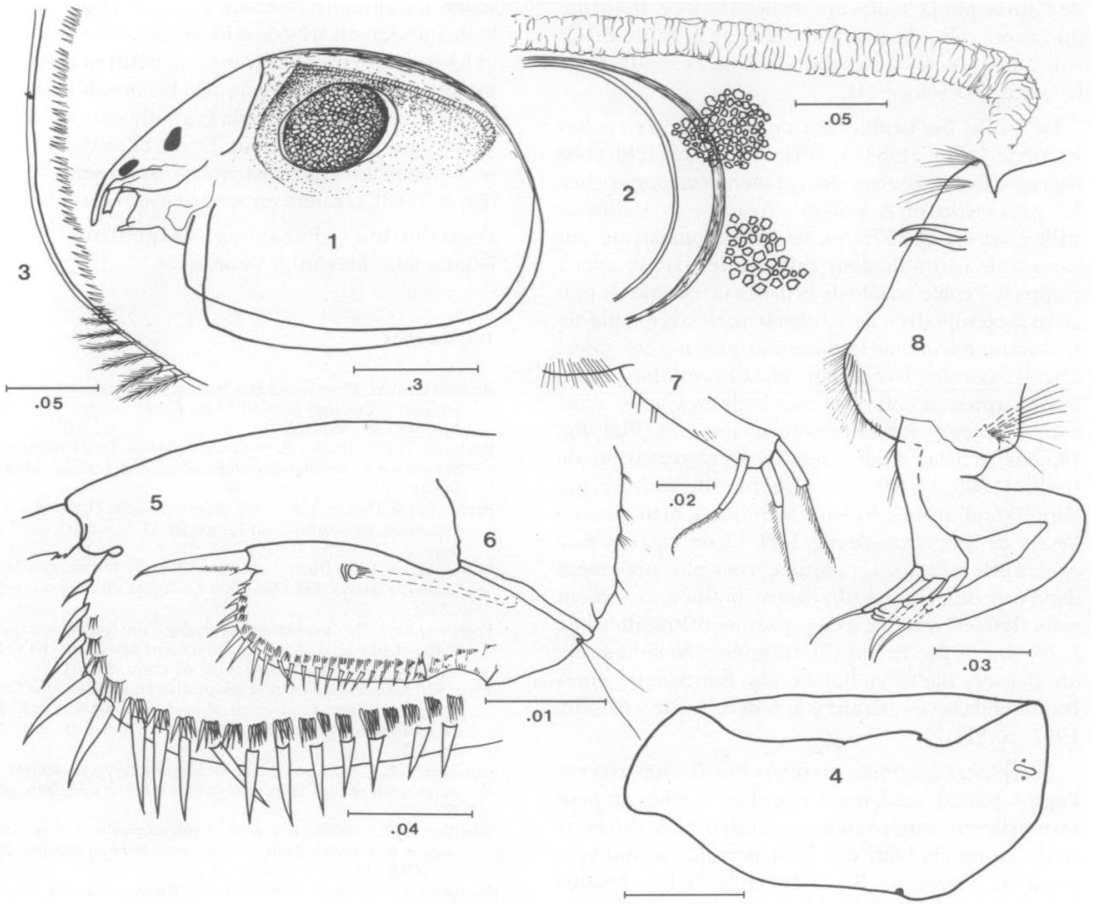

Planche III

Leydigiopsis omata Daday, 1905.

Fig. 1 à 3 : Femelle éphippiale. 1 : contour général et ephippium en vue latérale. 2 : détail de la structure et de la réticulation de l'ephippium. 3 : angle postéro-ventral de la valve gauche, face interne.

Fig. 4 à $8:$ Mâle. 4 : bouclier céphalique. $5:$ armature du postabdornen. 6 : postabdomen. $7:$ soies de la branche externe de l'endite de la patte thoracique I. 8 : patte thoracique I : corps de l'endite, branche externe de I'endite et clasper.

\section{3. - Discussion}

Des 4 espèces composant le genre Leydigiopsis, 3 espèces $L$. curvirostris, $L$. brevirostris, L. ornata sont maintenant connues de façon plus détaillée.

Pour Leydigiopsis megalops, rarement mentionnée dans la littérature, les données disponibles se rapportent essentiellement à la description de Sars (1901). Toutefois, la forme et la taille de l'ocelle (quadrangulaire et plus de 3 fois aussi grand que l'œil) ainsi que la forte indentation du bord antérieur du labre (Sars, op. cit. Pl. 8, fig. 12, 13.) suffisent à différencier $L$. megalops des autres espèces. du genre.

L. curvirostris se sépare d'emblée de $L$. brevirostris et $L$ ormata par la structure du rostre très allongé, recourbé ventralement, pouvant dépasser l'angle antéro-ventral des valves. 
Les 2 autres espèces peuvent être distinguées l'une de l'autre par la sculpture de la carapace, la forme du labre, celle du postabdomen, et la taille des 2 soies divergentes antérodorsales de l'exopodite de la patte thoracique III.

La taille de l'épine basale, par contre, selon Valdivia-Villar (1984) " difficilement visible chez notre espèce ( $L$. brevirostris) et bien développée chez $L$. curvirostris et $L$. ornata (traduit de ValdiviaVillar, op. cit., p. 1575), ne semble pas constituer un caractère véritablement différentiel. Nous avons comparé l'épine basale de la griffe terminale de plusieurs exemplaires de $L$. curvirostris avec celle de $L$. omata. Aucune différence marquée n'a été notée. Chez $L$. curvirostris comme chez $L$. ornata, l'épine basale apparaît courte et multifide en vue latérale, courte, large et fasciculée en vue dorsale. (Pl. I, fig. 14, cet article). Ceci corrobore l'observation de Daday (1905, p. 188) \& Die Nebenkralle ist kurz, am Hinterrand sitzen 2-3 kleine Härchen $n$, bien que son dessin de l'épine basale (fig. 3, Pl. 12, op. cit.) soit exagéré. Cette structure apparaît encore plus nettement dans une description ultérieure, malheureusement sans figures, relative à des spécimens brésiliens de L. ornata : « Die Endkralle trägt eine Nebenkamm, an dessen Basis vielleicht als Äquivalent eines Basalstachels - ein Kranz von Stacheln sitz "(Brehm 1937, p. 511).

Ainsi, chez $L$. arnata comme chez $L$. curvirosiris, l'épine basale, également peu développée, ne peut être prise en compte en tant que structure différentielle au moins pour ces 3 espèces. En ce qui concerne L. megalops, Sars (1901) ne figure aucune épine basale (fig. 14, pl. 18, op. cit.) et mentionne simplement dans sa description des griffes caudales finement denticulées.

Leydigiopsis omata a été récoltée dans la zone littorale de lacs d'inondation du système Orénoque. L'espèce habite probablement la couche supérieure des sédiments qu'elle fouit pour se nourrir. Sars (1901) observant ses élevages en aquarium, note que l'espèce ne fait que de très courtes incursions dans le milieu et se tient généralement sur le fond, plus ou moins profondément enfouie dans l'épaisseur des sédiments. La forme du corps, comprimé latéralement, les antennes munies d'épines et de spinules, le postabdomen très développé armé de nombreux et longs denticules, certaines structures comme les longues soies gnathobasiques, le coussinet de sétules de la face postérieure de la gnathobase de la patte thoracique II observé par Fryer chez des organismes colonisant des substrats mous ou semi-liquides (Fryer 1968), seraient en accord avec ce mode de vie.

Distribution: Paraguay, Argentine, Brésil, Guatemala, Mexique, Vénézuéla.

\section{Travaux cltes}

Brehm (V.) 1937. Brasilianische Cladoceren, gesammelt von Dr. O. Schubart. Zweiter Bericht. Int. Revie d. ges. Hydrob. a. Hydrogr., $35: 497.512$.

Bruhm (V.) 1938. Dritter Bericht über die von Dr. O. Schubart in Brasilien gesammelten Onychura. Zool. Anz Leipzig., 122, (3/4): 94103.

Brehm (V.) \& Thomsen (R.) 1936. Brasilianische Phyllopoden und Arguliden, gesammelt von Herrm Dr. O. Schubart. Zool Anz Leipzig, $116(7 / 8): 211.218$.

Daday (E.von) 1905 . Untersuchungen über die Süsswasser Mikrofauna Paraguays. VII. Cladocera Zoologica (Stuttgart)., 18, (44): 1-374.

Fryer (G.) 1963. The functional morphology and feeding mechanism of the chydorid cladoceran Eurycercus lamellatus (O.F. Müller). Trans, rov. Soc. Edinburgh, 65 (14): 355-381.

Fryer (G.) 1968. Evolution and adaptative radiation in the Chydoridae (Crustacea : Cladocera) : a study in comparative functional morphology and ecology. Phit. Trans. R. Soc, $(B), 254,(795)$ : 221-385.

Goulden (C.-E.) 1966a. La aguada de Santa Ana Vieja : an interpretative study of the Cladoceran fossils. Archiv. f. Hydrob., 62 (3): $373-404$

Goulden (C-E.) 1966b. The animal microfossils. The history of Laguna de Petenxil. Mem. Connecticut Acad. of Arts and Sciences, XVII : $84-120$

Martinez de Ferrato (A.) 1966. Nuevos Cladoceros para las aguas argentinas. I. Rev. Soc. Arg. Cienc. nas. (Buenos-Aires), Physis, XXVI (72) : $397-403$.

Rey (J.) \& Vasquez (E.) 1986. Cladocères de quelques corps d'eaux du bassin moyen de l'Orénuque (Vénézuela). Annls Limnol. 22 (2): 137-168.

Sars (G.O.) 1901 Contribution to the knowledge of the freshwater Entomostraca of South America, as shown by artificial hatching from dried material. Part I. Cladocera. Arch. Maith. Naturv: B., XXIII : 1-102.

Smirnov (N.-N) 1971. Chydoridae of the World's fauna. Fauna of the USSR, Crustacea, 1 (2), new series, $N^{\circ} 101$. Leningrad, 531 pp (in Russian). (English transl. A. Mercado., Israel prog. Sci. Transl., 1974).

Valdivia-Villar (R.) 1984. Sobre la morphologia de neotropicales crustaceos del genero Leydigiopsis (Cladocera, Chydoridae). Zoologisch. Joum., LXIII (10): 1572.1576. en russe

Zoppi de Roa (E.). Los Cladoceros de las sabanas inundables de Mantecal (Venezuela). Bol. Soc. Vent. Cienc. Nat (sous presse). 